\title{
Article
}

Elmeri Syrjänen, Stockholm University, Department of Psychology, SE-106 91, STOCKHOLM, SWEDEN

Email: elmeri.syrjanen@psychology.su.se

\section{Do Valenced Odors and Trait Body Odor Disgust Affect Evaluation of Emotion in Dynamic Faces?}

\section{Elmeri Syrjänen ${ }^{1}$, Marco Tullio Liuzza ${ }^{1,2}$, Håkan Fischer ${ }^{1}, \&$ Jonas K. Olofsson ${ }^{1,3}$}

${ }^{1}$ Stockholm University, Stockholm, Sweden

${ }^{2}$ Magna Græcia University, Catanzaro, Italy

${ }^{3}$ Swedish Collegium for Advanced Study, Uppsala, Sweden

\begin{abstract}
:
Disgust is a core emotion evolved to detect and avoid the ingestion of poisonous food as well as the contact with pathogens and other harmful agents. Previous research has shown that multisensory presentation of olfactory and visual information may strengthen the processing of disgust-relevant information. However, it is not known whether these findings extend to dynamic facial stimuli that changes from neutral to emotionally expressive, or if individual differences in trait body odor disgust may influence the processing of disgust-related information. In this pre-registered study, we tested whether a classification of dynamic facial expressions as happy or disgusted, and an emotional evaluation of these facial expressions, would be affected by individual differences in body odor disgust sensitivity (BODS), and by exposure to a sweat-like, negatively valenced odor (valeric acid), as compared to a soap-like, positively valenced odor (lilac essence) or a no-odor control. Using Bayesian hypothesis testing, we found evidence that odors do not affect recognition of emotion in dynamic faces even when BODS was used as moderator. However, an exploratory analysis suggested that an
\end{abstract}


unpleasant odor context may cause faster RTs for faces, independent of their emotional expression. Our results further our understanding of the scope and limits of odor effects on facial perception affect and suggest further studies should focus on reproducibility, specifying experimental circumstances where odor effects on facial expressions may be present vs absent.

Keywords: Disgust, Olfaction, Odors, Facial expression, Emotion, Reaction time, Evaluation

Rapidly decoding the facial expressions of other individuals is essential because faces convey important information about internal states and intentions (Palermo \& Rhodes, 2007; Rellecke, Sommer, \& Schacht, 2012). Previous research has shown that contextual information can inform the interpretation of facial expressions, see for example Wieser and Brosch (2012) for a comprehensive review. In the audio-visual domain contextual enhancement effects were shown for stimuli that are emotionally congruent, but also for emotional stimuli overall, see Gerdes, Wieser, and Alpers (2014) for review. Stimulus processing might be facilitated by congruent contextual information (Spence, 2007), but if the stimulus is emotional, unspecific arousal effects might also play a role (Pourtois, Schettino, \& Vuilleumier, 2013).

Apart from vision and hearing, olfaction is intimately linked to emotional processes (Croy et al., 2013; Croy, Olgun, \& Joraschky, 2011). Odors are associated with pleasant sensory experiences such as eating and drinking, but also with the avoidance of environmental hazards such as fire smoke and chemical pollution (Stevenson, 2010; Tybur, Lieberman, 
Kurzban, \& DeScioli, 2013). Odors may communicate emotional information such as disgust and fear (de Groot, Smeets, Kaldewaij, Duijndam, \& Semin, 2012), and perception of body odors may guide social preferences (Li, Moallem, Paller, \& Gottfried, 2007). Odors are particularly potent in evoking disgust, and disgust is the primary emotional response to unpleasant odors (Alaoui-Ismaïli, Robin, Rada, Dittmar, \& Vernet-Maury, 1997; AlaouiIsmaïli, Vernet-Maury, Dittmar, Delhomme, \& Chanel, 1997). In fact, odor-evoked disgust may be more resistant to top-down influences (Ferdenzi et al., 2013), and harder to suppress than disgust evoked by visual stimuli (Adolph \& Pause, 2012). Thus, odors might be particularly suitable stimuli for eliciting disgust reactions. In the present work, we investigate odor valence effects on the perception of disgusted and happy facial expressions.

Previous research showed that unpleasant odor contexts enhanced recognition accuracy for disgusted faces (Seubert, Kellermann, et al., 2010; Seubert, Loughead, et al., 2010). Leppanen and Hietanen (2003) found that disgusted expressions were recognized faster in an unpleasant odor context while happy expressions were recognized faster in a pleasant odor context. A recent study found that facial expressions were rated as more negative in an unpleasant odor context, and happy faces as more arousing in a pleasant odor context (Syrjänen et al., 2017). Furthermore, odors reduce the amount of emotional information that 
is needed to recognize a congruent facial expression (Leleu et al., 2015). These results would fit in a framework where congruent contextual stimuli facilitate the processing of facial expressions. In contrast, some prior work showed that valenced odors (i.e. pleasant or unpleasant) may result in unspecific enhanced recognition of disgusted or happy expressions (Seubert, Loughead, et al., 2010), while other showed that neutral faces are rated as more negative in a unpleasant odor context and more positive in a pleasant odor context (Cook et al., 2015). Perceiving sweat odor may increase the rated arousal of face stimuli, and sweat odors may enhance the early allocation of attentional processes in the structural encoding of faces (Adolph, Meister, \& Pause, 2013). Disgust-evoking images may influence vigilance that may lead to effective search patterns in pathogen que detection (Schienle, Ubel, Gremsl, Schongassner, \& Korner, 2016). These results indicate that the processing of pictures displaying facial expressions may be influenced by affective odors. In some of these studies there are specific congruency effects (Leleu et al., 2015; Leppanen \& Hietanen, 2003; Syrjänen et al., 2017); in others, general emotion effects are observed (Adolph et al., 2013; Cook et al., 2015; Seubert, Kellermann, et al., 2010; Seubert, Loughead, et al., 2010). Part of these differences might be explained by differences in study designs, however; individual differences in disgust responsivity might also play a key role in these studies. 
A recently developed scale, the Body Odor Disgust Scale, (BODS; Liuzza et al., 2016), effectively predicted disgust responses for human sweat odors (Liuzza, Olofsson, Sabiniewicz, \& Sorokowska, 2017), and was associated with perceived disease vulnerability (Liuzza et al., 2016). In many cultures, body odors are strong disgust elicitors (Curtis \& Biran, 2001) and, interestingly, people with olfactory malfunction are more prone to self-disgust (Ille, Wolf, Tomazic, \& Schienle, 2016). Not only do body odors contain disease-relevant information (Shirasu \& Touhara, 2011), humans can smell the presence of infectious diseases in others (Olsson et al., 2014), and infer personality traits of a person based on their body-odor (Sorokowska, Sorokowski, \& Szmajke, 2012). Thus, evidence suggests that disgust may be involved in social interactions, but that there are substantial individual differences in disgust propensity (Tybur, Lieberman, \& Griskevicius, 2009). While previous research in odor effects on facial perception has used static images, dynamic faces that changes from neutral to emotionally expressive might be regarded as more ecologically valid and more affective (Krumhuber, Kappas, \& Manstead, 2013; Sato \& Yoshikawa, 2007). Little is known about the effects on contextual odors on dynamic facial expressions. In the present study, we used facial expressions that morphed from 
neutral to either happy or disgusted expressions. To account for individual differences in body odor disgust, we used the BODS scale (Liuzza et al., 2016).

The results in (Seubert, Loughead, et al., 2010) suggested that any odor (vs. no odor condition) might facilitate the recognition speed of emotional expression, while the results in Leppanen and Hietanen (2003) indicated more specific odor congruency effects. Thus, we tested the following hypotheses:

- General arousing properties of odors affect recognition speed, which would lead to faster emotion recognition in the presence of an odor (vs. no odor condition), irrespective of odor-face emotional congruency.

- Emotions that are congruent with the valence of the odor are recognized faster (e.g., disgusted faces are recognized faster in an unpleasant odor context, whereas happy faces are recognized faster in a pleasant odor context).

Previous research has shown that valenced odors might affect rated valence and arousal of happy and disgusted expressions (Adolph et al., 2013; Cook et al., 2015; Syrjänen et al., 2017). Thus, we tested the hypothesis:

- Facial expressions are rated as more valenced and aroused when participants are exposed to an odor ( $v s$. no odor condition). 
We assessed participants scores on the BODS because of the intimate link between disgust and olfaction (Soudry, Lemogne, Malinvaud, Consoli, \& Bonfils, 2011; Zald \& Pardo, 1997), and the association between body odor disgust sensitivity and odor responses (Liuzza et al., 2016; Liuzza et al., 2017). The seemingly mixed findings in previous research might be resolved by considering individual differences in disgust sensitivity, which would lead to a better model fit. Our hypothesis was that high BODS score would be associated with greater emotional reactivity to disgust-related information.

- Participants with high BODS scores would rate disgusted faces as especially negatively valenced relative to happy and neutral faces.

- Participants with high BODS scores would generally rate facial expressions as being more negatively valenced in the no-odor and unpleasant odor conditions.

- High BODS scores would be associated with faster recognition of disgusted faces, relative to happy faces.

- Individual differences in BODS would moderate the decreased response latencies for disgusted faces during unpleasant odor context (i.e. individuals scoring high in BODS will drive the effect of reduced latencies to disgusted expressions). 
Because of the experimental design, we expected that these hypothesized results would not be explained by individual differences in perceived odor valence changes over the course of the experimental blocks (i.e. habituation).

Methods

\section{Sampling}

We used a Bayes Factor (BF) hypothesis-testing approach (Dienes, 2011) and we conducted a Sequential Bayes Factor (SBF) design, as proposed by Schonbrodt, Wagenmakers, Zehetleitner, and Perugini (2015). We defined a minimal sample size of $\mathrm{n}=$ 20 participants. However, we tested more participants until there was enough evidence for either the null hypothesis $\left(\mathrm{BF}_{01}>3\right.$, indicating "moderate evidence") or the alternative hypothesis $\left(\mathrm{BF}_{10}>6\right.$, "strong evidence", see planned analyses for more details). $\mathrm{A} \mathrm{BF}_{01}$ of 3 in support of the null hypothesis means that given the data, the null hypothesis is three times more likely than the alternative hypothesis, within the assumptions of the model. A $\mathrm{BF}_{10}$ of 6 for the alternative hypothesis was chosen because the choice of the default prior effect size distribution parameters in the BayesFactor package (Morey, Rouder, \& Jamil, 2017) can change the BF by a factor of two (Schonbrodt et al., 2015). Thus, a BF of 6 would ensure that, even in the worst-case scenario, evidence in favor of the alternative hypothesis would be at least moderate. The threshold for the null hypothesis was used to 
help us decide whether to stop sampling rather than making a strong inference on the null hypothesis per se.

Our maximum sample size was set to $n=70$ participants. This maximum sample size was determined through a Null Hypothesis Significance Testing (NHST) a priori power analysis for a medium effect size $(r=.3)$ and a one-tailed hypothesis, as we expected BODS to strengthen the effect of our experimental manipulation (unpleasant odor vs. neutral and pleasant odor) on the behavioral outcomes.

We recruited 21 adult participants $(8$ male) from the Stockholm University campus and online adverts (mean age 31.86 years, $\mathrm{SD}=10.93$ years). Participants did not report any severe cognitive, olfactory or visual deficits, allergies or neurological or psychiatric illness, and none of the participants reported being a daily smoker. Participants provided informed consent prior to participation and were informed that they were free to withdraw from participation at any time with full compensation. The study was conducted in accordance with the Declaration of Helsinki (1964).

\section{Surveys}

We collected demographic information in advance using the web-based program Qualtrics, along with measures of self-reported olfactory abilities, Right-Wing Authoritarianism scale 
(RWA, 15 items; Zakrisson, 2005), BODS (12 items; Liuzza et al., 2016), and self-reported placement on the liberal-conservative spectrum. The RWA and political orientation questionnaires were collected for a project unrelated to the present study and will be reported elsewhere. All questionnaires were presented in Swedish or in English (for native non-Swedish speakers).

\section{Stimuli}

We used the pictures of four male and four female faces (KDEF database; Lundqvist, Flykt, \& Öhman, 1998), each displaying neutral, happy and disgusted facial expressions. Using these pictures, we created sixteen unique video clips lasting three seconds each. In each video clip, a neutral facial expression develops gradually into either a happy or a disgusted expression. The video clips were created by morphing neutral expressions (starting point) with disgusted and happy expressions of the same individual (endpoint includes 50\% of expressions). Stimulus parameters were determined by pilot testing (where emphasis was put to enhance the effective range during each trial where the facial expression is ambiguous) using the MorphX freeware program (http://www.norrkross.com/software/morphx/download.php). All pictures and video clips

were presented with the PsychoPy presentation software (v1.83.04; Peirce, 2007) on a LCD-screen and covered a horizontal visual angle of $8.5^{\circ}$ and a vertical angle of $13.5^{\circ}$. 
The participants were tested under three different conditions: No odor, lilac odor (Stockholms Eter \& Essencefabrik), or valeric acid (Sigma-Aldrich). The unpleasant (valeric acid) and pleasant (lilac essence) odors were carefully diluted with a nearly odorless solution (Propylene glycol, 1,2-propanediol 99\%, Sigma-Aldrich). Valeric acid has been used as a substitute for human sweat in previous research (Bensafi, 2002; de Araujo, Rolls, Velazco, Margot, \& Cayeux, 2005), and is a specific compound in human sweat associated with disease (Shirasu \& Touhara, 2011). Lilac odor is commonly used in a variety of settings (Surburg \& Panten, 2016).

Based on previous psychophysical investigations $(n=60)$, we used a concentration of $30 \%$ for both odors, which represents a matched moderate intensity (Syrjänen et al., 2017). In short, in the previous study participants rated both odorants in 3 concentrations, $1 \%, 40 \%$, and $80 \%$, for valence (positive and negative) and intensity on the Borg CR-100 scale (Borg $\&$ Borg, 2002). From these intensity ratings, the odor concentration was estimated to match a moderate intensity ( 25 on the Borg scale) by using a linear interpolation. In the present study, odor exposure was achieved through placing odorized cotton pads in an elastic cotton tube that was attached under the nose of participants, as in our previous study (Syrjänen et al., 2017). A cotton pad containing only the near-odorless solvent was used in the "no odor" condition. All subjects were exposed to all three odor conditions, and the 
order of odor exposure, as well as the order of stimuli within each block, was fully randomized.

\section{Procedure}

The participants performed an emotion recognition task that consisted of categorizing dynamic facial expressions as either "disgusted" or "happy". The sixteen unique 3-second video clips were displayed three times each in a block. Across three blocks, each lasting about 5 minutes, the task was carried out under odor exposure in three conditions (pleasant odor, unpleasant odor, no odor). At the beginning and end of each block, the participants rated the pleasantness of the odor. At the end of each block, the participants rated the valence and arousal of the emotions expressed by each of the three facial stimulus types (i.e. neutral expression, $50 \%$ disgusted, and 50\% happy) for each of the eight actors.

\section{Analysis}

To account for response accuracy we calculated d-prime scores with methods suggested by Macmillan and Creelman (2009), by subtracting the z-transformed hit rates from the $\mathrm{z}$ transformed false-alarm rates for each participant and condition. Because some participants 
performed at $100 \%$ accuracy we subtracted .5 points from the hits and added .5 points to the false-alarms.

We used the $1 \mathrm{mBF}$ (linear models) function in the BayesFactor package in $\mathrm{R}$ to calculate Bayes Factors (BF) for each of our hypotheses. In each statistical test we considered participants and actors expressing the emotion as random effects. To test for moderating effects of individual disgust sensitivity we added BODS score as an interaction term to these analyses (see http://osf.io/2bje7for specific analytic strategy). We interpreted the sizes of BFs according to the recommendations of Raftery (1995) as referred to by (Jarosz \& Wiley, 2014).

The Cauchy prior distribution for the parameter under alternative defines our prior beliefs about the possible effect sizes and provides a good alternative to a completely uninformative prior. In addition, it has some desirable properties (namely, scale invariance, consistency and consistency in information) that motivate its use in the BayesFactor $\mathrm{R}$ package (Rouder, Morey, Speckman, \& Province, 2012). A Cauchy prior has fatter tails than a normal, thus allowing for more extreme observations. Defining a Cauchy prior scaling factor allows to have more or less diffuse prior beliefs on the parameters under the alternative. We conducted the same analysis with the default-scaling factor for the Cauchy prior distribution: medium $(r=0.5)$, wide $(r=0.702)$ and ultra-wide $(\mathrm{r}=1)$. Following the 
current standards, we use subscripts on Bayes factors to refer to the models compared (i.e. $\mathrm{BF}_{10}$ denotes the comparison of the alternative with the null model, and $\mathrm{BF}_{01}$ the null against the alternative model). Accordingly, the Bayes factor for the alternative relative to the null is denoted $\mathrm{BF}_{10}$, while the Bayes factor for the null relative to the alternative is denoted $\mathrm{BF}_{01}$.

Results

\section{Manipulation checks}

The participants rated the odor valence at the start and end of each block. There was very strong evidence that the odor valence ratings differed predictably between odors, such that the valeric acid odor was indeed rated as more unpleasant than the lilac odor, $\mathrm{BF}_{10}>150$. We wanted to ensure that habituation effects could not explain the findings for the Reaction Times (RTs) and ratings, and we found positive evidence that the odors were rated similarly at the beginning and end of each block $\mathrm{BF}_{01}=5.05$, and that this effect was not moderated by BODS score $\mathrm{BF}_{01}=6.98$. We calculated the mean valence ratings for each odor and each participant at the beginning and end of each block. The rated difference between unpleasant odor and no odor was $-41.38(\mathrm{SD}=23.31)$ units on the valence scale 
$\left(\mathrm{BF}_{10}>150\right)$. The rated difference between pleasant odor and no odor was $12.55(\mathrm{SD}=$ 19.47) on the odor valence scale $\left(\mathrm{BF}_{10}=6.17\right)$. Lastly, there was very strong evidence that the pleasant and unpleasant odor differed in rated odor valence, $\left(\mathrm{BF}>150_{10}\right)$, differing by $53.93(\mathrm{SD}=21.82)$ scale units.

We calculated credible intervals for the arousal and valence ratings by sampling from the posterior distribution (100,000 iterations) for each main effect separately (i.e., expression and odor, see Figure 1). For arousal there was very strong evidence $\mathrm{BF}_{10}>150$ for a difference in ratings (i.e. rated arousal: disgusted > happy > neutral). For valence, as expected, disgusted faces were rated as more negative than neutral, and happy faces were rated as more positive in comparison to neutral faces $\left(\mathrm{BF}_{10}>150\right)$. Similar credible intervals for odor effects on expression ratings were all overlapping. 


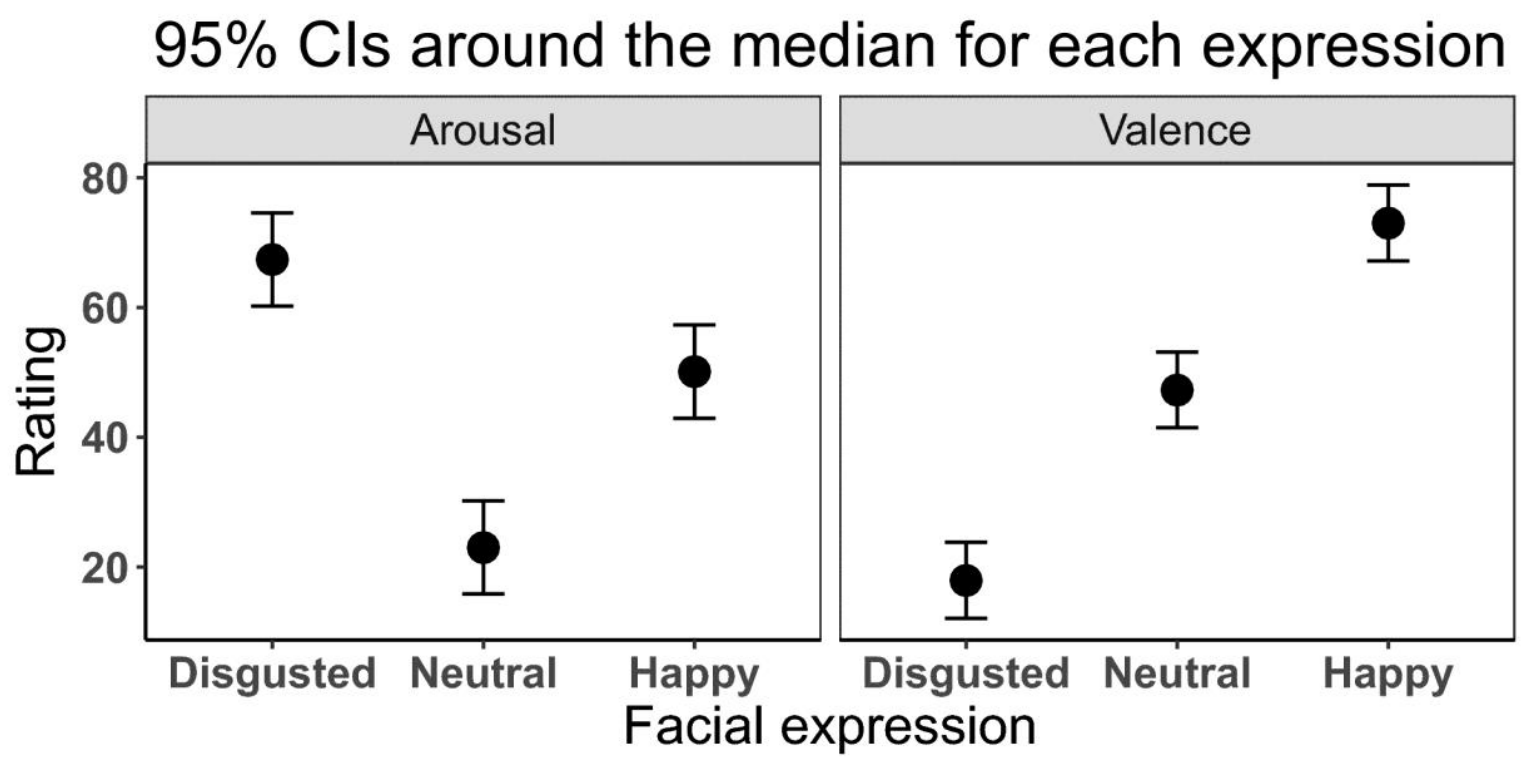

Figure 1. The left panel shows the credible intervals around the median for the arousal ratings (VAS scale 1-100) of each facial expression (n.b., increasing numbers indicate higher arousal). The right panel shows the credible intervals around the median for the rated valence of the facial expressions. Note that low values indicate negative valence in relation to higher values.

To account for differences in accuracy between the conditions we performed a 3 by 2 Bayesian ANOVA (3 levels of odor vs. 2 levels of expression). The main effects model containing only expression was strongly preferred against the other models $\left(\mathrm{BF}_{10}>150\right)$, indicating that the d-prime score for happy expressions $(\mathrm{M}=3.15, \mathrm{SD}=0.73)$ was higher than the d-prime score for disgusted expressions $(\mathrm{M}=2.46, \mathrm{SD}=1.19)$. To illustrate this 
effect (Figure 2.), we calculated 95\% credible intervals around the median accuracy using similar methods as above. To account for individual variability in performance we added participant as a random factor, and this model was strongly preferred $\left(\mathrm{BF}_{10}>150\right)$ in comparison with the non-random factor model, indicating that response accuracy varied between individuals.

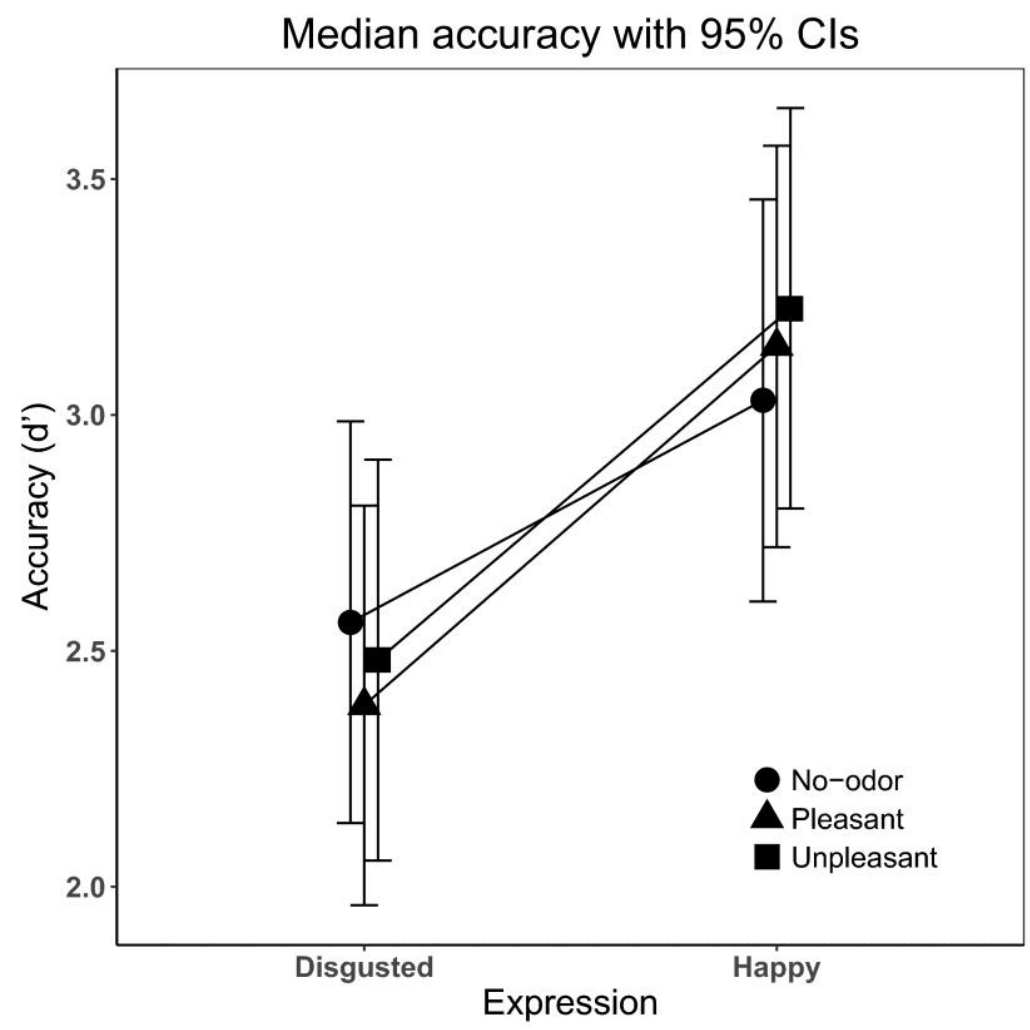

Figure 2. Accuracy (dPrime) for each expression and odor with credible intervals around the median. 


\section{Odor effects}

We tested whether there were general odor effects on recognition of facial expressions. We found positive evidence for the null with a Bayes Factor $\left(\mathrm{BF}_{01}\right)$ of 4.69 (i.e., odor in general did not affect emotion recognition speed; see Table 1. for mean RTs and SDs for each condition).

We assessed if there were specific congruency effects between the odor stimuli and facial expressions. The results showed that there was very strong evidence for the null with a $\mathrm{BF}_{01}$ of 107.55 (i.e. congruency between odor and facial expression did not decrease the recognition speed).

Lastly, we assessed how the odors affected the subjective properties of the faces. For arousal ratings, there was positive evidence for the null $\mathrm{BF}_{01}=14.30$, (i.e., facial expressions were not rated as more arousing in the context of an odor). Similarly, for valence, there was very strong evidence against odor affecting valence ratings $\mathrm{BF}_{01}=45.52$ (see Table 1. for mean (SD) arousal and valence ratings). 
Table 1.

Mean and (SD) for each condition and measure. Note that the behavioral task included faces that changed from neutral to disgusted and happy expressions, reaction time and accuracy data were not collected for neutral expressions. Accuracy is presented as d-prime values.

\begin{tabular}{lcccc}
\hline \multicolumn{1}{c}{ Odor/Expression } & $\underline{\mathrm{RT}}$ & $\underline{\text { Accuracy }}$ & $\underline{\text { Valence }}$ & $\underline{\text { Arousal }}$ \\
Pleasant/Happy & $1809(635)$ & $3.17(0.71)$ & $73.41(16.99)$ & $48.49(22.28)$ \\
Pleasant/Neutral & & & $47.46(12.25)$ & $23.30(20.04)$ \\
Pleasant/Disgusted & $1823(831)$ & $2.34(1.12)$ & $17.88(14.79)$ & $67.92(18.37)$ \\
Neutral/Happy & $1774(666)$ & $3.01(0.69)$ & $72.62(16.39)$ & $51.58(19.77)$ \\
Neutral/Neutral & & & $47.49(13.74)$ & $21.70(19.17)$ \\
Neutral/Disgusted & $1905(790)$ & $2.58(1.27)$ & $17.42(12.54)$ & $67.18(18.44)$ \\
Unpleasant/Happy & $1760(599)$ & $3.26(0.80)$ & $73.04(16.31)$ & $50.25(22.70)$ \\
Unpleasant Neutral & & & $46.86(13.79)$ & $23.82(19.20)$ \\
Unpleasant/Disgusted & $1791(793)$ & $2.46(1.22)$ & $18.88(15.00)$ & $67.33(17.53)$ \\
\hline
\end{tabular}




\section{Effects of individual differences}

We assessed if these null results could be explained by individual differences in trait body odor disgust. For the interaction between BODS score and reaction time, we found that there was positive evidence for the null $\mathrm{BF}_{01}=9.01$ (i.e. BODS score did not predict faster reaction times for disgusted facial expressions).

For the specific BODS moderation of reaction times for unpleasant stimuli, there was strong support that BODS did not moderate RTs for recognition of disgusted expressions in the disgusting odor condition $\mathrm{BF}_{01}=55.95$.

Similarly, for valence ratings, there was positive evidence for the null, $\mathrm{BF}_{01}=8.54$, that BODS score was not related to more negative evaluation of disgusted faces.

Lastly, there was strong evidence against a possible moderating role of the BODS on the valence ratings in the unpleasant and no-odor conditions $\mathrm{BF}_{01}=34.61$.

\section{Exploratory analyses}

Although results from the planned analyses generated evidence against our pre-registered hypotheses, we conducted further exploratory analyses. Here, we reasoned that because an 
unpleasant odor is a pathogen cue, it might facilitate emotion recognition overall. We found a slight advantage in the unpleasant odor context, a main effects analysis contrasting unpleasant odor context versus no-odor and pleasant odor contexts showed weak evidence $\mathrm{BF}_{10}=2.87$ in favor for an effect of unpleasant odor on response speed (i.e. in unpleasant odor condition, faces were recognized faster, regardless of expression). We computed the credible intervals around the median for each condition by sampling from the posterior distribution (100,000 iterations) for each main effect separately (i.e., expression and odor). The left-hand panel in Figure 3. shows the effect of unpleasant odor.

We conducted a further exploratory analysis to investigate the effect of facial expression on reaction times. In particular, we investigated whether happy faces are recognized faster than disgusted faces, as shown previously in the literature (Palermo \& Coltheart, 2004). As the right-hand panel in Figure 3. suggests there was an advantage in recognizing happy expressions, we followed up this effect by performing similar Bayesian linear model analysis as previously but modeling only the main effect of expression as an independent variable. The analysis indicated strong evidence for a difference in reaction time, $\mathrm{BF}_{10}>$ 150 (i.e. happy expressions were recognized faster than disgusted). 


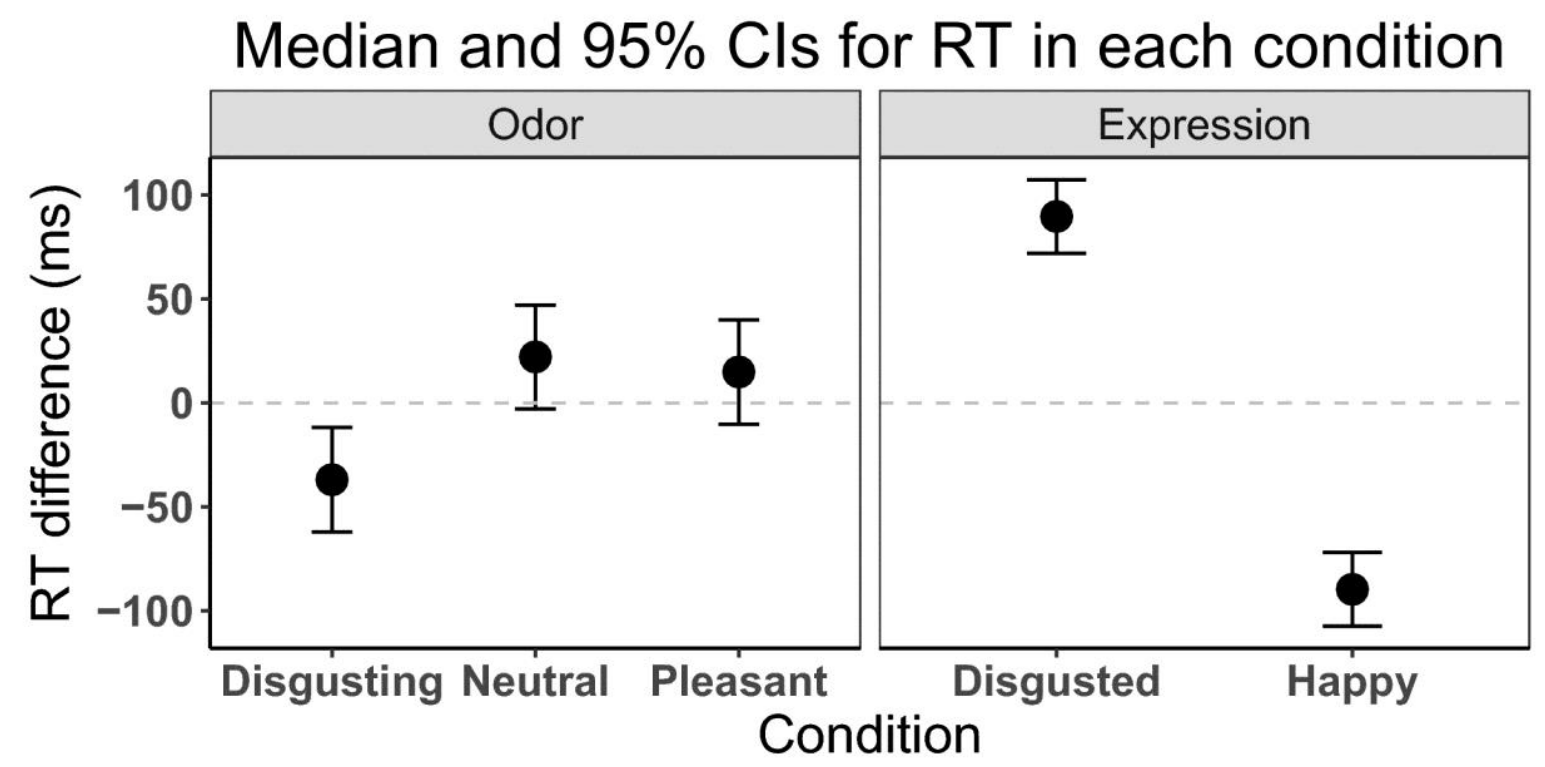

Figure 3. Credible intervals (Cls) around the median, separately for main effects of odor (left side), and expression (right side) on reaction times. Note that the medians and Cls for RTs reflect the difference from grand mean (indicated by the gray dashed line) for each condition.

As odor valence is subjective, we also performed the analyses for the pre-registered hypotheses with odor valence treated as a continuous variable based on participant ratings. The results, however, were unchanged. 


\section{Discussion}

Previous research indicates that valenced odors generally seem to affect recognition of static emotional facial expressions (Leleu et al., 2015; Seubert, Kellermann, et al., 2010; Seubert, Loughead, et al., 2010), however, some studies also show specific effects for emotionally congruent odor-face stimuli (Adolph et al., 2013; Leppanen \& Hietanen, 2003). Little is known about how odors affect dynamic facial emotion expressions, which are arguably more ecologically relevant. In the present study, we used dynamic faces that changed from neutral to either disgusted or happy expressions in a $3 \mathrm{~s}$ video clip, in the context of pleasant odor, no odor, or unpleasant odor. Our hypotheses were pre-registered and based on the prior literature. Results showed no effects of odor on either emotion recognition or affective evaluations. Our results also indicated that these null findings could not be explained by individual differences in body odor disgust sensitivity. Results of manipulation checks suggested that our evidence for the null hypotheses could not be attributed to a failed odor manipulation.

Specifically, we found positive evidence of no overall effects of odors on the response speed in emotion recognition for dynamic faces. The results, furthermore, provided strong evidence against the notion that the congruency between odor valence and facial expression determined the response speed. These results contrast with previous research on static 
images of faces, resulting in generic effects of odors (Seubert, Kellermann, et al., 2010; Seubert, Loughead, et al., 2010), or specific congruency effects (Leppanen \& Hietanen, 2003) on reaction times. We found strong support for the notion that happy faces were recognized faster in comparison to disgusted expressions, extending previous results of happy face advantage for static images (Palermo \& Coltheart, 2004). Whereas, previous research has used static images of affective faces, the present study used dynamic expressions. In light of the results, there is a distinct possibility that odor effects on facial emotion recognition do not generalize to dynamic faces. The results in Seubert, Kellermann, et al. (2010), indicate that the effect of facial expression is much larger than the effect of odor. The present findings suggest that there might be ceiling effects, such that dynamic faces are more arousing (Krumhuber et al., 2013), and there might not be room for the arousing properties of odors to further affect reaction times. Another possibility is that the task was too easy, potentially resulting in only visual information being attended to. However, ceiling level performance levels cannot explain the lack of odor effects. First, task difficulty varied between conditions, and accuracy was higher for happy faces (replicating the main effect in Leppanen and Hietanen (2003), study 2). Second, similarly, happy faces were recognized faster than disgusted expressions (replicating the main effect in Leppanen and Hietanen (2003), study 2). Third, a model with participant as a random factor provided a better explanation of the difference in accuracy than a model without, 
indicating that individuals varied in how difficult the task was. Thus, performance did not reach ceiling-levels.

The results in prior literature suggested that odors would increase the rated arousal and valence of the facial expression in comparison to the no-odor condition. Instead, we found positive to strong evidence that for the arousal and valence ratings, there were no effects of odor. We note that the previously reported results for static images are not entirely consistent (Adolph et al., 2013; Cook et al., 2015; Syrjänen et al., 2017). The present study differed in some aspects compared to previous studies that investigated odor effects on ratings, the present study and Syrjänen et al. (2017) used valeric acid as unpleasant odors whereas Adolph et al. (2013) used human sweat, and, Cook et al. (2015) used methyl mercaptan. This might indicate that different odors may be more or less effective in affecting social perceptions. Also, the affective properties were measured with different scales, Adolph et al. (2013) and Syrjänen et al. (2017) used the SAM rating procedure, whereas; the present study and Cook et al. (2015) used VAS scales. As the SAM rating scale is designed to measure subjective affective reactions toward emotional stimuli (Bradley \& Lang, 1994), it might be better at capturing internal states that odors are particularly effective at eliciting in comparison to other stimuli (Adolph \& Pause, 2012). In the present study we did not collect odor intensity ratings, but pre-selected odors of 
'moderately strong' intensity. While it might be argued that odors need to reach a high intensity threshold before affecting the affective properties of faces or that these effects are affected by habituation, that would also necessarily exclude most odors present in socially relevant situations. However, previous studies have shown that for odors, intensity and valence ratings are highly correlated (Distel, 1999), so it is unlikely that perceived odor intensity decreased significantly across our experiment, as such habituation would have resulted also in more neutral valence ratings over time (which was not the case). In fact, several prior studies using similar odor exposures and found that neither intensity ratings nor valence ratings decreased significantly over time (Adolph \& Pause, 2012; Anderson et al., 2003). Habituation is thus a highly unlikely reason for our results.

Could the lack of odor effects be explained by individual differences in disgust sensitivity? Taking BODS scores into account, we found strong evidence against a moderating effect of body odor disgust sensitivity on reaction times. Although we did not use actual body odors in the present study, valeric acid were used extensively in the literature as a body-odor related smell (e.g. Anderson et al., 2003), and it is associated with actual odors emitted by the body (Doty, 1981; Shirasu \& Touhara, 2011). Although valeric acid has been associated with cheese in a previous study (i.e. de Araujo et al., 2005), that study tested a mixture of cheese and iso-valeric acid in order to make the odor resemble cheese. 
We conducted exploratory analyses to find that the unpleasant smell reduced the time needed to recognize facial expressions overall. This topic is not well explored in the literature, and thus not part of the preregistered hypotheses, but the effect is in line with the reasoning that unpleasant odors act as pathogen cues (Tybur et al., 2013), and it might make the organism generally more attentive to visual cues in the environment (Adolph et al., 2013; Steinberg et al., 2012).

Taken together, the previously reported effects of odor on emotional face perception might not generalize to dynamic faces. More research is needed on the influence of valenced odors on static as well as dynamic face stimuli. We note that our present study presents some strength in relation to the previous research. In line with recent concerns about the soundness of psychological research (Nosek et al., 2015), the present study was preregistered and we will publish all data and other relevant material (i.e. experimental files and analysis code). We also used Bayesian statistical methods that permitted evidence in favor of the null hypotheses. Methods such as pre-registration are designed to discourage practices that are harmful for science, such as "Hypothesizing After the Results are Known" (HARKing; Kerr, 1998), and the publication of all used data and analysis scripts will help to establish reproducible results and future meta-analyses (Open Science Collaboration, 2015). 
In summary, in this pre-registered study we show that pleasant and unpleasant odors did not affect the time needed to recognize happy or disgusted dynamic facial expressions. We also show that these odors did not affect how happy, neutral and disgusted facial expressions were rated on neither valence, nor arousal. Lastly, we could demonstrate that these effects were not moderated by individual differences in body odor disgust. The slight effect from our exploratory analysis, that unpleasant odor lowered overall response times, may help guide researchers to conclusive results in future lines of inquiry, preferably using Bayesian statistics and pre-registered analysis plans.

\section{Acknowledgements}

We would like to thank Elena Ubaldini for assistance with data collection.

\section{Funding}

This research was supported by funding from the Swedish Foundation for the Humanities and Social Sciences (M14-0375:1), and Marianne and Marcus Wallenberg Foundation (MMW 2014:0178) to JKO and by a research grant to MTL from the Swedish Research Council (2016-02018 


\section{References}

Adolph, D., Meister, L., \& Pause, B. M. (2013). Context counts! social anxiety modulates the processing of fearful faces in the context of chemosensory anxiety signals. Front Hum Neurosci, 7, 283. doi:10.3389/fnhum.2013.00283

Adolph, D., \& Pause, B. M. (2012). Different time course of emotion regulation towards odors and pictures: are odors more potent than pictures? Biol Psychol, 91(1), 65-73. doi:10.1016/j.biopsycho.2012.05.003

Alaoui-Ismaïli, O., Robin, O., Rada, H., Dittmar, A., \& Vernet-Maury, E. (1997). Basic Emotions Evoked by Odorants. Physiol Behav, 62(4), 713-720. doi:10.1016/s0031-9384(97)90016-0

Alaoui-Ismaïli, O., Vernet-Maury, E., Dittmar, A., Delhomme, G., \& Chanel, J. (1997). Odor Hedonics: Connection With Emotional Response Estimated by Autonomic Parameters. Chem Senses, 22(3), 237-248. doi:10.1093/chemse/22.3.237

Anderson, A. K., Christoff, K., Stappen, I., Panitz, D., Ghahremani, D. G., Glover, G., . . Sobel, N. (2003). Dissociated neural representations of intensity and valence in human olfaction. Nature neuroscience, 6(2), 196-202. doi:10.1038/nn1001

Bensafi, M. (2002). Autonomic Nervous System Responses to Odours: the Role of Pleasantness and Arousal. Chem Senses, 27(8), 703-709. doi:10.1093/chemse/27.8.703

Borg, E., \& Borg, G. (2002). A comparison of AME and CR100 for scaling perceived exertion. Acta Psychologica, 109(2), 157-175. doi:10.1016/s0001-6918(01)00055-5

Bradley, M. M., \& Lang, P. J. (1994). Measuring emotion: The self-assessment manikin and the semantic differential. J Behav Ther Exp Psychiatry, 25(1), 49-59. doi:10.1016/00057916(94)90063-9

Cook, S., Fallon, N., Wright, H., Thomas, A., Giesbrecht, T., Field, M., \& Stancak, A. (2015). Pleasant and Unpleasant Odors Influence Hedonic Evaluations of Human Faces: An Event-Related Potential Study. Front Hum Neurosci, 9, 661. doi:10.3389/fnhum.2015.00661

Croy, I., Laqua, K., Suss, F., Joraschky, P., Ziemssen, T., \& Hummel, T. (2013). The sensory channel of presentation alters subjective ratings and autonomic responses toward disgusting stimuli-Blood pressure, heart rate and skin conductance in response to visual, auditory, haptic and olfactory presented disgusting stimuli. Front Hum Neurosci, 7, 510. doi:10.3389/fnhum.2013.00510

Croy, I., Olgun, S., \& Joraschky, P. (2011). Basic emotions elicited by odors and pictures. Emotion, 11(6), 1331-1335. doi:10.1037/a0024437

Curtis, V., \& Biran, A. (2001). Dirt, Disgust, and Disease: Is Hygiene in Our Genes? Perspectives in Biology and Medicine, 44(1), 17-31. doi:10.1353/pbm.2001.0001

de Araujo, I. E., Rolls, E. T., Velazco, M. I., Margot, C., \& Cayeux, I. (2005). Cognitive modulation of olfactory processing. Neuron, 46(4), 671-679. doi:10.1016/j.neuron.2005.04.021 
de Groot, J. H., Smeets, M. A., Kaldewaij, A., Duijndam, M. J., \& Semin, G. R. (2012). Chemosignals communicate human emotions. Psychol Sci, 23(11), 1417-1424.

doi:10.1177/0956797612445317

Dienes, Z. (2011). Bayesian Versus Orthodox Statistics: Which Side Are You On? Perspect Psychol Sci, 6(3), 274-290. doi:10.1177/1745691611406920

Distel, H. (1999). Perception of Everyday OdorsCorrelation between Intensity, Familiarity and Strength of Hedonic Judgement. Chem Senses, 24(2), 191-199. doi:10.1093/chemse/24.2.191

Doty, R. L. (1981). Olfactory communication in humans. Chem Senses, 6(4), 351-376. doi:10.1093/chemse/6.4.351

Ferdenzi, C., Roberts, S. C., Schirmer, A., Delplanque, S., Cekic, S., Porcherot, C., . . Grandjean, D. (2013). Variability of affective responses to odors: culture, gender, and olfactory knowledge. Chem Senses, 38(2), 175-186. doi:10.1093/chemse/bjs083

Gerdes, A. B., Wieser, M. J., \& Alpers, G. W. (2014). Emotional pictures and sounds: a review of multimodal interactions of emotion cues in multiple domains. Front Psychol, 5, 1351. doi:10.3389/fpsyg.2014.01351

Ille, R., Wolf, A., Tomazic, P. V., \& Schienle, A. (2016). Disgust-Related Personality Traits in Men with Olfactory Dysfunction. Chem Senses, 41(5), 427-431. doi:10.1093/chemse/bjw017

Jarosz, A. F., \& Wiley, J. (2014). What Are the Odds? A Practical Guide to Computing and Reporting Bayes Factors. Journal of Problem Solving, 7(1). doi:10.7771/1932-6246.1167

Kerr, N. L. (1998). HARKing: hypothesizing after the results are known. Pers Soc Psychol Rev, 2(3), 196-217. doi:10.1207/s15327957pspr0203_4

Krumhuber, E. G., Kappas, A., \& Manstead, A. S. R. (2013). Effects of Dynamic Aspects of Facial Expressions: A Review. Emotion Review, 5(1), 41-46. doi:10.1177/1754073912451349

Leleu, A., Demily, C., Franck, N., Durand, K., Schaal, B., \& Baudouin, J. Y. (2015). The Odor Context Facilitates the Perception of Low-Intensity Facial Expressions of Emotion. PLoS One, 10(9), e0138656. doi:10.1371/journal.pone.0138656

Leppanen, J. M., \& Hietanen, J. K. (2003). Affect and face perception: odors modulate the recognition advantage of happy faces. Emotion, 3(4), 315-326. doi:10.1037/15283542.3.4.315

Li, W., Moallem, I., Paller, K. A., \& Gottfried, J. A. (2007). Subliminal smells can guide social preferences. Psychol Sci, 18(12), 1044-1049. doi:10.1111/j.1467-9280.2007.02023.x

Liuzza, M. T., Lindholm, T., Hawley, C., Sendén, M. G., Ekström, I., Olsson, M. J., . . O Olofsson, J. K. (2016). The Body Odor Disgust Scale (BODS): Development and Validation of a Novel Olfactory Disgust Assessment. Chem Senses, bjw107. doi:10.1093/chemse/bjw107

Liuzza, M. T., Olofsson, J. K., Sabiniewicz, A., \& Sorokowska, A. (2017). Body odor trait disgust sensitivity predicts perception of sweat bio-samples. Chem Senses.

doi:10.1093/chemse/bjx026 
Lundqvist, D., Flykt, A., \& , \& Öhman, A. (1998). The Karolinska directed emotional faces (KDEF): CD ROM from Department of Clinical Neuroscience, Psychology section, Karolinska Institutet.

Macmillan, N. A., \& Creelman, C. D. (2009). Detection theory: A user's guide: Psychology press.

Morey, R. D., Rouder, J. N., \& Jamil, T. (2017). BayesFactor Package (Version 0.9.9).

Nosek, B. A., Alter, G., Banks, G. C., Borsboom, D., Bowman, S. D., Breckler, S. J., . . Yarkoni, T. (2015). Promoting an open research culture. Science, 348(6242), 1422-1425. doi:10.1126/science.aab2374

Olsson, M. J., Lundstrom, J. N., Kimball, B. A., Gordon, A. R., Karshikoff, B., Hosseini, N., . . . Lekander, M. (2014). The scent of disease: human body odor contains an early chemosensory cue of sickness. Psychol Sci, 25(3), 817-823. doi:10.1177/0956797613515681

Open Science Collaboration. (2015). Estimating the reproducibility of psychological science. Science, 349(6251), aac4716. doi:10.1126/science.aac4716

Palermo, R., \& Coltheart, M. (2004). Photographs of facial expression: Accuracy, response times, and ratings of intensity. Behavior Research Methods, Instruments, \& Computers, 36(4), 634638. doi:10.3758/bf03206544

Palermo, R., \& Rhodes, G. (2007). Are you always on my mind? A review of how face perception and attention interact. Neuropsychologia, 45(1), 75-92. doi:10.1016/j.neuropsychologia.2006.04.025

Peirce, J. W. (2007). PsychoPy--Psychophysics software in Python. J Neurosci Methods, 162(1-2), 813. doi:10.1016/j.jneumeth.2006.11.017

Pourtois, G., Schettino, A., \& Vuilleumier, P. (2013). Brain mechanisms for emotional influences on perception and attention: what is magic and what is not. Biol Psychol, 92(3), 492-512. doi:10.1016/j.biopsycho.2012.02.007

Rellecke, J., Sommer, W., \& Schacht, A. (2012). Does processing of emotional facial expressions depend on intention? Time-resolved evidence from event-related brain potentials. Biol Psychol, 90(1), 23-32. doi:10.1016/j.biopsycho.2012.02.002

Rouder, J. N., Morey, R. D., Speckman, P. L., \& Province, J. M. (2012). Default Bayes factors for ANOVA designs. Journal of Mathematical Psychology, 56(5), 356-374. doi:10.1016/j.jmp.2012.08.001

Sato, W., \& Yoshikawa, S. (2007). Enhanced Experience of Emotional Arousal in Response to Dynamic Facial Expressions. J Nonverbal Behav, 31(2), 119-135. doi:10.1007/s10919-0070025-7

Schienle, A., Ubel, S., Gremsl, A., Schongassner, F., \& Korner, C. (2016). Disgust Proneness and the Perception of Disgust-Evoking Pictures An Eye-Tracking Experiment. Journal of Psychophysiology, 30(3), 124-129. doi:10.1027/0269-8803/a000162 
Schonbrodt, F. D., Wagenmakers, E. J., Zehetleitner, M., \& Perugini, M. (2015). Sequential Hypothesis Testing With Bayes Factors: Efficiently Testing Mean Differences. Psychol Methods. doi:10.1037/met0000061

Seubert, J., Kellermann, T., Loughead, J., Boers, F., Brensinger, C., Schneider, F., \& Habel, U. (2010). Processing of disgusted faces is facilitated by odor primes: a functional MRI study. Neuroimage, 53(2), 746-756. doi:10.1016/j.neuroimage.2010.07.012

Seubert, J., Loughead, J., Kellermann, T., Boers, F., Brensinger, C. M., \& Habel, U. (2010). Multisensory integration of emotionally valenced olfactory-visual information in patients with schizophrenia and healthy controls. J Psychiatry Neurosci, 35(3), 185-194. doi:10.1503/jpn.090094

Shirasu, M., \& Touhara, K. (2011). The scent of disease: volatile organic compounds of the human body related to disease and disorder. J Biochem, 150(3), 257-266. doi:10.1093/jb/mvr090

Sorokowska, A., Sorokowski, P., \& Szmajke, A. (2012). Does Personality Smell? Accuracy of Personality Assessments Based on Body Odour. European Journal of Personality, 26(5), 496-503. doi:10.1002/per.848

Soudry, Y., Lemogne, C., Malinvaud, D., Consoli, S. M., \& Bonfils, P. (2011). Olfactory system and emotion: common substrates. Eur Ann Otorhinolaryngol Head Neck Dis, 128(1), 18-23. doi:10.1016/j.anorl.2010.09.007

Spence, C. (2007). Audiovisual multisensory integration. Acoustical Science and Technology, 28(2), 61-70. doi:10.1250/ast.28.61

Steinberg, C., Dobel, C., Schupp, H. T., Kissler, J., Elling, L., Pantev, C., \& Junghofer, M. (2012). Rapid and highly resolving: affective evaluation of olfactorily conditioned faces. Journal of Cognitive Neuroscience, 24(1), 17-27. doi:10.1162/jocn_a_00067

Stevenson, R. J. (2010). An initial evaluation of the functions of human olfaction. Chem Senses, 35(1), 3-20. doi:10.1093/chemse/bjp083

Surburg, H., \& Panten, J. (2016). Common fragrance and flavor materials: preparation, properties and uses. Weinheim, Germany: John Wiley \& Sons.

Syrjänen, E., Wiens, S., Fischer, H., Zakrzewska, M., Wartel, A., Larsson, M., \& Olofsson, J. K. (2017). Cortical integration of odor-visual emotional signals in the N170 component. Submitted for publication.

Tybur, J. M., Lieberman, D., \& Griskevicius, V. (2009). Microbes, mating, and morality: individual differences in three functional domains of disgust. J Pers Soc Psychol, 97(1), 103-122. doi:10.1037/a0015474

Tybur, J. M., Lieberman, D., Kurzban, R., \& DeScioli, P. (2013). Disgust: evolved function and structure. Psychol Rev, 120(1), 65-84. doi:10.1037/a0030778

Wieser, M. J., \& Brosch, T. (2012). Faces in context: a review and systematization of contextual influences on affective face processing. Front Psychol, 3, 471.

doi:10.3389/fpsyg.2012.00471 
Zakrisson, I. (2005). Construction of a short version of the Right-Wing Authoritarianism (RWA) scale. Personality and Individual Differences, 39(5), 863-872. doi:10.1016/j.paid.2005.02.026

Zald, D. H., \& Pardo, J. V. (1997). Emotion, olfaction, and the human amygdala: Amygdala activation during aversive olfactory stimulation. Proceedings of the National Academy of Sciences, 94(8), 4119-4124. doi:10.1073/pnas.94.8.4119 